\title{
BMJ Open Management of type 2 diabetes in China: the Happy Life Club, a pragmatic cluster randomised controlled trial using health coaches
}

Colette Browning, ${ }^{1,2,3}$ Anna Chapman, ${ }^{1,2}$ Hui Yang, ${ }^{2,3}$ Shuo Liu, ${ }^{4}$ Tuohong Zhang, ${ }^{5}$ Joanne C Enticott, ${ }^{1,2,6}$ Shane A Thomas ${ }^{2,3}$

To cite: Browning $\mathrm{C}$, Chapman $\mathrm{A}$, Yang $\mathrm{H}$, et al. Management of type 2 diabetes in China: the Happy Life Club, a pragmatic cluster randomised controlled trial using health coaches. BMJ Open 2016;6:e009319. doi:10.1136/bmjopen-2015009319

- Prepublication history and additional material is available. To view please visit the journal (http://dx.doi.org/ 10.1136/bmjopen-2015009319).

Received 6 July 2015 Revised 9 February 2016 Accepted 10 February 2016

CrossMark

For numbered affiliations see end of article.

Correspondence to

Anna Chapman;

anna.chapman@monash.edu

\section{ABSTRACT}

Objective: To assess the effectiveness of a coach-led motivational interviewing (MI) intervention in improving glycaemic control, as well as clinical, psychosocial and self-care outcomes of individuals with type 2 diabetes mellitus (T2DM) compared with usual care.

Design: Pragmatic cluster randomised controlled trial (RCT).

Setting: Community Health Stations (CHSs) in Fengtai district, Beijing, China.

Participants: Of the 41 randomised CHSs (21 intervention and 20 control), 21 intervention CHSs (372 participants) and 18 control CHSs (296 participants) started participation.

Intervention: Intervention participants received telephone and face-to-face MI health coaching in addition to usual care from their CHS. Control participants received usual care only. Medical fees were waived for both groups.

Outcome measures: Outcomes were assessed at baseline, 6 and 12 months. Primary outcome measure was glycated haemoglobin (HbA1c). Secondary outcomes included a suite of anthropometric, blood pressure (BP), fasting blood, psychosocial and selfcare measures.

Results: At 12 months, no differential treatment effect was found for HbA1c (adjusted difference 0.02, 95\% $\mathrm{Cl}-0.40$ to $0.44, p=0.929$ ), with both treatment and control groups showing significant improvements. However, two secondary outcomes: psychological distress (adjusted difference $-2.38,95 \% \mathrm{Cl}-4.64$ to $-0.12, p=0.039$ ) and systolic BP (adjusted difference $-3.57,95 \% \mathrm{Cl}-6.08$ to $-1.05, \mathrm{p}=0.005$ ) were robust outcomes consistent with significant differential treatment effects, as supported in sensitivity analyses. Interestingly, in addition to $\mathrm{HbA1c}$, both groups displayed significant improvements in triglycerides, LDL cholesterol and HDL cholesterol.

Conclusions: In line with the current Chinese primary healthcare reform, this study is the first large-scale cluster RCT to be implemented within real-world CHSs in China, specifically addressing T2DM. Although a differential treatment effect was not observed for $\mathrm{HbA1C}$, numerous outcomes (including HbA1c) improved in both groups, supporting the establishment

\section{Strengths and limitations of this study}

- This is a rigorous pragmatic cluster randomised controlled trial implemented in real-world Community Health Stations (CHSs) in urban China, utilising existing resources.

- The pragmatic nature of this trial meant that data collection was conducted by multiple data collectors from multiple CHSs and resulted in higher than anticipated levels of missing data.

- Medication use and adherence was not rigorously assessed, however, it can be assumed that both groups were medicated similarly as per the Chinese Diabetes Society guideline.

of regular, free clinical health checks for people with T2DM in China.

Trial registration number: ISRCTN01010526; Preresults.

\section{INTRODUCTION}

China has become the global leader in the number of diabetes mellitus (DM) cases, and this is a critical and costly issue. The International Diabetes Federation estimated that, in 2015, there were 109.6 million DM cases in China, and this number is predicted to rise to 150.7 million by $2040 .{ }^{1}$ China's DM prevalence is higher in older age groups, in urban residents and in individuals living in prosperous regions. ${ }^{12}$ The WHO estimated DM-related costs to China for 2005-2015 to be US $\$ 558$ billion. ${ }^{3}$ Effective and efficient DM management and prevention are therefore key goals for China.

China is in the midst of major healthcare reforms intended to build a communitybased primary health system to enable patients to access health services for basic and less costly healthcare. ${ }^{4}$ In order to manage the large numbers of DM cases, the 
Chinese government has advocated best practice medical management. ${ }^{5}{ }^{6}$ For T2DM, the guideline focuses on long-term management, shifting from a hospital-based model, towards delivery in primary healthcare settings. ${ }^{7}$ This approach requires the training of primary healthcare doctors and nurses in new ways to support their patients to manage their T2DM.

The use of coach training, behaviour change and counselling approaches for T2DM is widespread in Western countries. However, these approaches have not been widely tested in China. Ismail and colleagues' (2004) admittedly now dated-meta-analysis of psychological interventions for the management of $\mathrm{T}^{2} \mathrm{DM}^{8}$ identified only two Chinese studies, one utilising cognitivebehavioural therapy techniques, which found improvements in psychological status, ${ }^{9}$ and the other using supportive psychotherapy, finding improvements in glycaemic control and weight. ${ }^{10} \mathrm{Fu}$ and Dong's ${ }^{11}$ meta-analysis of behavioural and psychological approaches to the management of patients with DM with depression or anxiety symptoms in China found some evidence that such approaches improved depressive symptoms and blood glucose control.

A large body of research literature suggests unassisted behaviour change programmes rarely work effectively in chronic disease management. ${ }^{12}$ However, equally, it cannot be assumed that healthcare workers have the requisite skills to provide effective support. Most healthcare workers require behaviour change training to assist patients to achieve their goals. Motivational interviewing (MI) is a widely used technique in such programmes, and this was used in the current reported trial. ${ }^{13}$ Miller and Rollnick define MI as "a collaborative, goal-oriented style of communication with particular attention to the language of change. It is designed to strengthen personal motivation for and commitment to a specific goal by eliciting and exploring the person's own reasons for change within an atmosphere of acceptance and compassion" (ref 13, p.29). The exploration and resolution of ambivalence is a major focus of MI, and it is considered a particularly useful technique for individuals who are ambivalent to change a particular behaviour. ${ }^{13}$

In preparatory work, we piloted health coach training for doctors and nurses in MI in Fangzhuang, Beijing. Subsequently, we developed this full trial to test the effectiveness of the intervention. ${ }^{14}$ The pilot showed significant improvements in glycated haemoglobin (HbA1c) levels at 6 months. ${ }^{15}$

In this paper, we report a pragmatic cluster randomised controlled trial (RCT) of a T2DM management intervention using health coaches in a primary healthcare setting in Beijing, China. We hypothesised that, compared to usual care, a structured coach training approach delivered within a pragmatic study design would enhance the ability of patients to self-manage T2DM, leading to improved clinical and psychological outcomes, thus delaying diabetic complications. In addition, we anticipated beneficial impacts on components of metabolic syndrome and the risk factors for cardiovascular disease.
METHODS

\section{Study design and participants}

This pragmatic cluster RCT was implemented from December 2011 to December 2013, in the Fengtai District of Beijing, China, which, in 2012, had a population of 2.2 million residents. ${ }^{16}$ A cluster RCT design was chosen to minimise contamination between the control and intervention participants that may occur if participants in the same health service are randomised to different treatment arms. Further, because the intervention was targeted at changing the behaviour of health professionals, once trained, treatment leakage could have occurred because it would be have been difficult for them to avoid using the intervention techniques with control participants. The selection of a pragmatic trial design, which is undertaken in the 'real world' and with usual care, ${ }^{17}$ also suited the context of the intervention site, namely Community Health Stations (CHSs) within a district of Beijing where preventive care, health management, primary medical care, rehabilitation, health education and family planning are offered. ${ }^{18}$ Additionally, the intention of this pragmatic trial was to assist in supporting a decision on whether to deliver the chosen intervention as part of routine care. ${ }^{17}$

In this instance, CHSs were chosen as the cluster unit and all government run CHSs within the Fengtai district were deemed as eligible. This resulted in an original total cluster sample size of 42 CHSs. Consent for CHS participation was sought before randomisation, directly from the Fengtai Health Bureau, which govern all eligible CHSs. Prior to CHS (cluster) randomisation, an independent person at each participating CHS identified eligible participants from the patient records maintained at each site. Patients with T2DM were eligible to participate if they were aged 50 years and over, lived in the Fengtai district, had an established health record and were receiving care at one of the participating CHSs. Exclusion criteria included the inability to understand and provide informed consent (eg, people with cognitive problems), or a medical condition that precluded adherence to recommendations (eg, end stage cancer). No further exclusion criteria were implemented due to the pragmatic nature of this trial. Once generated, each CHS submitted the participant lists for stratification and random sampling.

Sample size estimates were calculated using the fixed number of clusters available to the current study $(\mathrm{k}=42)$. The intracluster correlation coefficient (ICC) accounts for the greater similarity of responses of patients within clusters compared with between clusters, and we applied an ICC of 0.05 as typical in primary care settings. ${ }^{19}$ The effect size for primary outcome, HbAlc, was anticipated to be 0.32 , as determined from a published meta-analysis. ${ }^{19}$ Using these parameters, a sample size calculator for cluster-randomised trials ${ }^{19}$ estimated a total sample of $n=726$ would be required to achieve at 
least $80 \%$ power, while maintaining an $\alpha$ of $5 \%$ and a participant attrition rate of $20 \%$.

The above sample size reflected an average of 18 patients per CHS (cluster); however, the number of eligible patients varied greatly between CHSs, necessitating CHSs to be categorised into small and large stations, with different targets imposed for the numbers of patients to be recruited. Small CHSs were defined as stations with less than 80 eligible patients and were asked to recruit 15 patients, while large stations of over 80 patients were asked to recruit 25 patients per station. As a result, the total number of patients targeted for recruitment became 780 (395 intervention group and 385 control group). Figure 1 shows the flow of CHSs and participants through the trial.

\section{Stratification and randomisation}

Clusters (CHSs) were stratified by geographical location (outer, central and inner) in order to achieve a balance of groups. The sociodemographic profile of residents in Fengtai varies according to geographic location. The geographic distribution is a combination of longitude (west, central and east) and radiation from city centre (2nd, 3rd and 4th ring roads), such that outer Fengtai is predominantly a regional/rural area; central Fengtai is a residential suburban area; and inner Fengtai is a developed urban area. CHSs were randomised into the control or intervention groups, using block randomisation. In order to minimise selection bias, this process was carried out centrally by an independent person and all CHSs were coded to ensure the randomisation was a

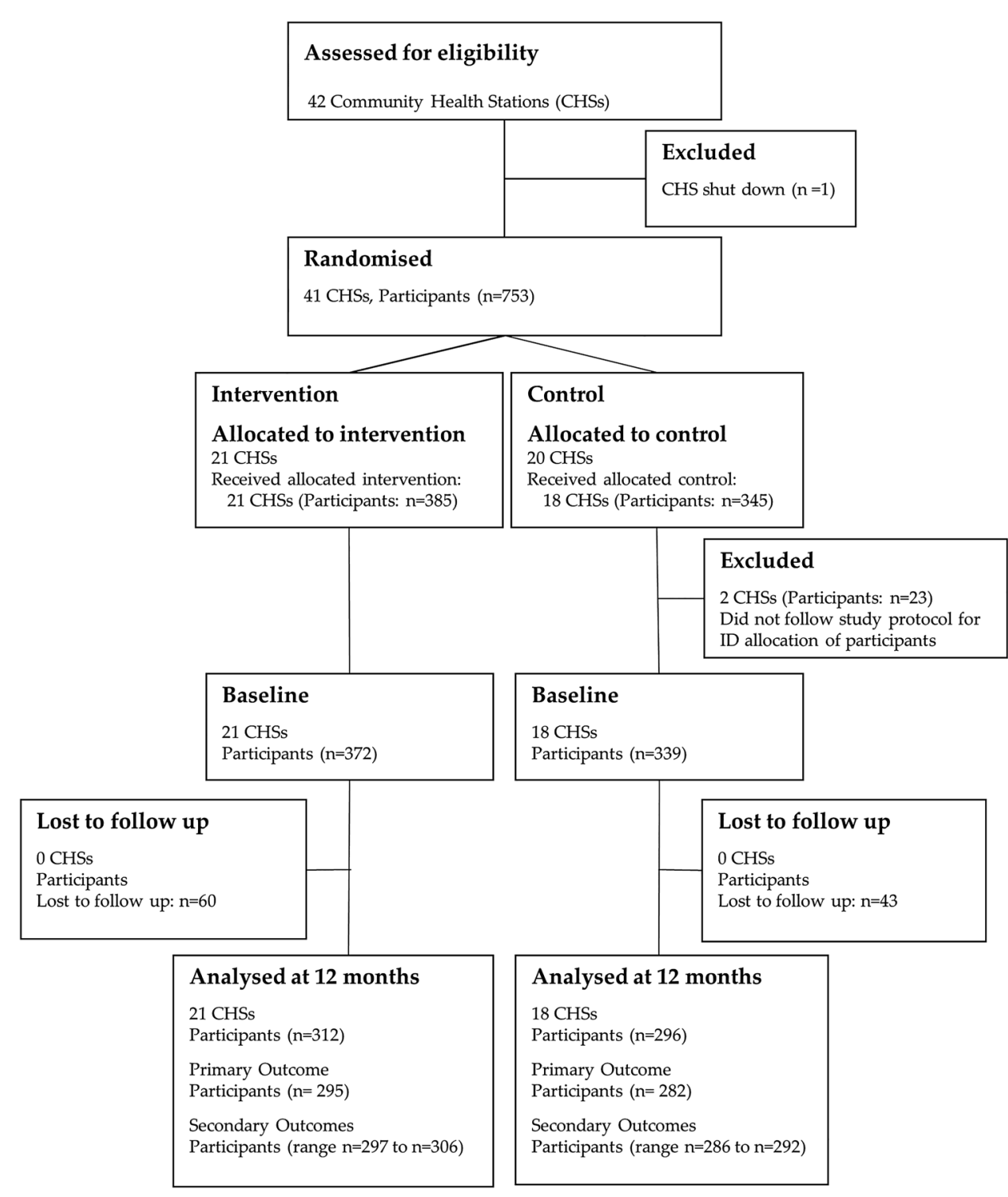

Figure 1 CONSORT flow diagram of Community Health Stations (CHSs) and participants through trial. Of the original $41 \mathrm{CHSs}$ (21 intervention CHS and 20 control CHS), 2 control CHSs did not follow study protocol with regard to participant recruitment and baseline data collection, which resulted in incorrect ID allocation, and were therefore removed from analysis. This resulted in a total of 21 intervention CHSs and 18 control CHSs included in the study analyses. 
blinded process. Prior to CHS randomisation, one CHS was permanently shut down, resulting in 41 CHSs randomised into the study (21 intervention CHSs and 20 control CHSs). To ensure the required sample size was still achieved following this closure, one neighbouring CHS recruited double the participant numbers (ie, 30 instead of 15).

Participants were sampled by computerised random allocation software that stratified by gender in order to achieve balance. The sampling procedure produced an ordered list of eligible participants; CHS recruiting officers systematically invited eligible participants into the study. If a potential participant was unable to be contacted via telephone on the first attempt, a further two attempts were made before classifying that participant as 'not contactable'. Once a participant declined participation or was classified as not contactable, the recruiting officer moved to the next person on the list. All participants were provided with an explanatory statement and consent form, either by mail or in person at their next CHS visit.

\section{Outcomes}

The primary outcome measure was HbA1c. Secondary clinical outcomes included systolic and diastolic blood pressure (BP), weight, body mass index (BMI), waist and hip circumference, as well as fasting blood samples (fasting plasma glucose, total cholesterol, triglyceride, high-density lipoprotein (HDL) and low-density lipoprotein (LDL) ) and were assessed as per the published study protocol, ${ }^{14}$ with the exception of LDL cholesterol, which was measured using a direct method. Additionally, secondary psychosocial and self-care behaviour outcomes included: psychological distress (Kessler 10 (K10); score range $10-50),{ }^{20}$ quality of life (QoL) (WHO QoL-BREF; domain score range $0-100),{ }^{21}$ diabetes self-care activities (SDSCA; score range $0-7$, representing number of days/ week) ${ }^{22}$ and diabetes management self-efficacy (C-DMSES; score range 0-200). ${ }^{23}$ See online supplementary material file for a full description of the psychosocial and self-care measures.

All patient level outcomes were assessed at baseline, and again at 6 and 12 months, during a clinical health check and an interviewer-administered questionnaire. All participants were instructed to fast overnight for a minimum of $8 \mathrm{~h}$, and participant fasting times were recorded prior to each blood test. Where fasting times were not sufficient, participants were asked to reschedule their appointment. Intervention and control groups both underwent the same assessments, and all participants were informed of their clinical results. Participants who did not present for outcome assessment were followed up whenever possible by phone call and asked to reschedule.

Owing to the pragmatic nature of this trial, data collectors were not blinded to group allocation; however, laboratory technicians were blinded. Blood samples were analysed centrally at the Fengtai Center for Disease
Control and Prevention Laboratory, and certified in external quality assurance by the National Center for Clinical Laboratories of China.

\section{Procedure}

As recommended for RCTs utilising complex interventions, ${ }^{24}$ figure 2 graphically depicts the treatment conditions for intervention as well as for control groups.

Control group participants received usual care from their CHS as outlined in the Chinese Guideline for Diabetes Prevention and Management. ${ }^{7}$ While usual care is typically expected to vary in pragmatic trials between patients and providers, and according to institutional policies, the Chinese DM guideline recommends patients attend quarterly doctor consultations and complete an annual physical examination. The guideline also includes referral to DM specialists, traditional Chinese medicine physicians and physiotherapists. In order to complete the data collection requirements for the study, increased monitoring that deviated from usual care recommendations was necessary.

Intervention group participants received a combination of telephone and face-to-face health coaching in addition to usual care from their CHS. Health coaching was performed by experienced clinicians (community doctors, nurses and psychologists) from each CHS. Prior to starting the intervention, health coaches completed a certified training programme in coach-assisted chronic disease management. This consisted of a preworkshop learning phase, which included the study of key concepts in patient-centred communications, health psychology, epidemiology of key targeted illnesses and conditions, MI and behaviour change, programme evaluation, clinical outcome measurement and the Happy Life Club intervention protocol. ${ }^{14}$ This was followed by a 2-day intensive MI workshop that introduced health coaches to the framework and rationale of MI, and included the application of MI core skills across the behaviour change process. To ensure health coaches were supported in their MI skill development, refresher workshops were also conducted throughout the intervention. One month after the intervention began, the coaches received a further half day advanced training workshop and were contacted every 3 months for debriefing and feedback.

Health coaches aimed to assist participants in achieving the treatment targets as outlined in the Chinese Guideline for Diabetes Prevention and Management, ${ }^{7}$ with the primary goal of treatment of HbAlc of less than $7.0 \%$. An intervention manual that utilised existing local guidelines and recommendations (eg, Dietary Guidelines for Chinese Residents) was used to guide health coaches. ${ }^{25}$ Owing to the pragmatic nature of the trial, it was anticipated that health coaches would adapt the intervention manual to their local contexts and, as such, variation in intervention delivery was expected.

The initial step in each health coaching session was to set the agenda for the session with the participant. This 


\begin{tabular}{|c|c|c|c|}
\hline \multicolumn{2}{|c|}{ Timeline } & $\begin{array}{l}\text { MI Health Coaching } \\
\text { (Intervention) }\end{array}$ & $\begin{array}{l}\text { Usual Care } \\
\text { (Control) }\end{array}$ \\
\hline \multicolumn{4}{|c|}{ Randomisation } \\
\hline \multicolumn{2}{|l|}{ Baseline } & \multicolumn{2}{|c|}{ Measurement of outcomes } \\
\hline \multicolumn{4}{|c|}{ 0-3 month (per month) } \\
\hline \multicolumn{4}{|c|}{ 3-6 month (per month) } \\
\hline \multicolumn{2}{|l|}{6 month } & \multicolumn{2}{|c|}{ Measurement of outcomes } \\
\hline \multicolumn{4}{|c|}{ 6-12 month (per month) } \\
\hline \multicolumn{2}{|c|}{12 month } & \multicolumn{2}{|c|}{ Measurement of outcomes } \\
\hline (a) & \multicolumn{3}{|c|}{ Telephone call made by health coach trained in MI } \\
\hline (b) & \multicolumn{3}{|c|}{ Face-to-face session with health coach trained in MI } \\
\hline
\end{tabular}

Figure 2 Graphical depiction of interventions for each treatment condition. MI, motivational interviewing.

was achieved by asking the participant to identify the most productive place to start the conversation, that is, 'What would be helpful to talk about today?'. Once the participant identified a key issue for discussion, health coaches utilised their complex set of MI skills by assessing current behaviour in relation to the issue, and determined where the participant was in the change process. Health coaches then guided the conversation with the ultimate aim of strengthening the participants' own motivation and commitment for change. By stepping out of the expert role into a more guiding, collaborative role, health coaches engaged the person in the process of making significant, lasting changes in their own life.

In the first 3 months, participants received two face-to-face and two telephone coaching sessions per month. The frequency of sessions decreased over the 12-month intervention period such that in the last 6 months of the intervention, participants received one face-to-face and one telephone session per month (figure 2). This reflects the philosophy of the MI approach whereby, over time, input diminishes as the participants gain confidence in self-management.

In neither treatment group did participants receive payment for participation in this study, however, medical fees (for both, consultation and out-of-pocket pathology costs) associated with participation in the project were waived, with associated costs absorbed by the CHSs. Although China has near universal health insurance coverage, individuals with T2DM typically incur out-of-pocket expenses for both, medical (consultation and pathology costs) and pharmaceutical care. ${ }^{26}$

\section{Statistical analysis}

Descriptive statistics were used to summarise characteristics of CHSs and participants, with regard to baseline characteristics and patterns of mean change over time. To account for clustering and repeated measures collected from participants, we used multilevel mixed effects models with robust SEs, implemented with xtmixed, using Stata software (V.12). Random effects accounted for within-site clustering and within-patient correlation. Fixed effects included time and intervention group. The primary analysis examined 6 and 12-month changes for HbA1c. Secondary analyses included all clinical, psychosocial and self-care continuous outcomes. Model fit was examined by comparing AIC values.

Models were adjusted for baseline variables of age group, gender, chronic comorbidity category, time since T2DM diagnosis, highest education level and residential address socioeconomic status (categories listed in tables 1 and 2). ICCs were estimated using one way analysis of variance. A level of $\mathrm{p}<0.05$ was used to evaluate statistical significance of all outcomes.

A missing data analysis was carried out for each outcome measure and consisted of Little's MCAR test to investigate patterns of missingness in variables of group allocation (intervention/control) and baseline characteristics as listed in table 3. Sensitivity analyses were carried out using multiple imputation to account 
Table 1 Biochemical and clinical outcomes at baseline, and 12 months, by study group

\section{Intervention group}

\section{Control group}

Available case analysis

\section{N Mean \pm SD}

N Mean \pm SD

Adjusted difference between

\section{Outcomes}

bA1c (\%)

Baseline

12 months

Mean change from baseline to 12 months $(95 \% \mathrm{CI})$

Secondary outcomes

Weight $(\mathrm{kg})$

Baseline

12 months

Mean change from baseline to 12 months $(95 \% \mathrm{Cl})$

BMl $\left(\mathrm{kg} / \mathrm{m}^{2}\right)$

Baseline

12 months

Mean change from baseline to 12 months $(95 \% \mathrm{Cl})$

Waist $(\mathrm{cm})$ men

Baseline

12 months

Mean change from baseline to 12 months $(95 \% \mathrm{Cl})$

Waist $(\mathrm{cm})$ women

Baseline

12 months

Mean change from baseline to 12 months $(95 \% \mathrm{Cl})$

Hip (cm) men

Baseline

12 months

Mean change from baseline to 12 months $(95 \% \mathrm{Cl})$

Hip (cm) women

Baseline

12 months

Mean change from baseline to 12 months $(95 \% \mathrm{Cl})$

Systolic blood pressure $(\mathrm{mm} \mathrm{Hg})$

Baseline

12 months

Mean change from baseline to 12 months $(95 \% \mathrm{CI})$

Diastolic blood pressure $(\mathrm{mm} \mathrm{Hg})$

Baseline

12 months

Mean change from baseline to 12 months $(95 \% \mathrm{Cl})$
$359 \quad 10.60+2.09$

$6.67 \pm 1.69$

$-3.86(-4.16$ to -3.56$)$

$359-70.13 \pm 11.71$

$30370.10 \pm 11.74$

$+0.13(-0.15$ to 0.41$)$

$359 \quad 26.23 \pm 3.69$

$302 \quad 26.22 \pm 3.70$

$+0.05(-0.05$ to 0.16$)$

$93.75 \pm 9.29$

$92.21 \pm 9.36$

$-0.88(-1.48$ to -0.27$)$

$186 \quad 88.57 \pm 9.57$

$163 \quad 88.14 \pm 9.16$

$-0.05(-0.69$ to 0.59$)$

$176 \quad 102.37 \pm 8.69$

$140 \quad 102.40+9.08$

$+0.11(-0.52$ to 0.74$)$

$18699.95 \pm 8.23$

$162100.19 \pm 8.27$

$+0.31(-0.32$ to 0.94$)$

$363 \quad 129.03 \pm 15.14$

$305 \quad 127.79+11.71$

$-1.57(-3.17$ to 0.04$)$

$363 \quad 76.91 \pm 9.10$

$30577.46 \pm 7.05$

$+0.17(-0.72$ to 1.06$)$
$323 \quad 10.29 \pm 1.71$

$282 \quad 6.64 \pm 1.59$

$-3.69(-3.93$ to -3.44$) \quad 0.29 \quad 0.02(-0.40$ to 0.44$)$

0.929

$333 \quad 69.68 \pm 10.27$

$292 \quad 69.51 \pm 9.97$

$+0.32(0.06$ to 0.58$) \quad 0.07 \quad-0.09(-0.58$ to 0.40$)$

0.723

$333 \quad 26.03 \pm 3.42$

$292 \quad 26.13 \pm 3.44$

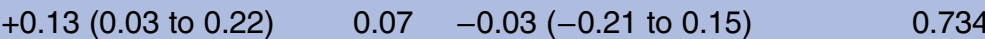

$153 \quad 92.82 \pm 8.49$

$124 \quad 92.85 \pm 8.07$

$+0.56(-0.04$ to 1.15$) \quad 0.20-1.28(-2.46$ to -0.11$) \quad 0.032$

$176 \quad 90.68 \pm 10.02$

$167 \quad 90.90 \pm 9.20$

$+0.32(-0.33$ to 0.97$) \quad 0.17 \quad-0.92(-1.95$ to 0.11$) \quad 0.080$

$153 \quad 100.23 \pm 7.71$

$125 \quad 100.54 \pm 7.25$

$+0.92(0.18$ to 1.66$) \quad 0.23 \quad-0.03(-1.34$ to 1.29$) \quad 0.967$

$179 \quad 102.06 \pm 8.81$

$166 \quad 102.41 \pm 8.78$

$+0.55(-0.06$ to 1.16$) \quad 0.22 \quad-0.36(-1.58$ to 0.85$) \quad 0.557$

$334 \quad 128.46 \pm 14.82$

$292 \quad 130.96 \pm 12.37$

$+2.40(0.89$ to 3.91$) \quad 0.07 \quad-3.57(-6.08$ to -1.05$) \quad 0.005 \ddagger$

$334 \quad 76.00 \pm 8.64$

$29276.95 \pm 7.23$

$+1.04(0.00$ to 2.08$) \quad 0.13 \quad-0.50(-2.18$ to 1.18$)$ 


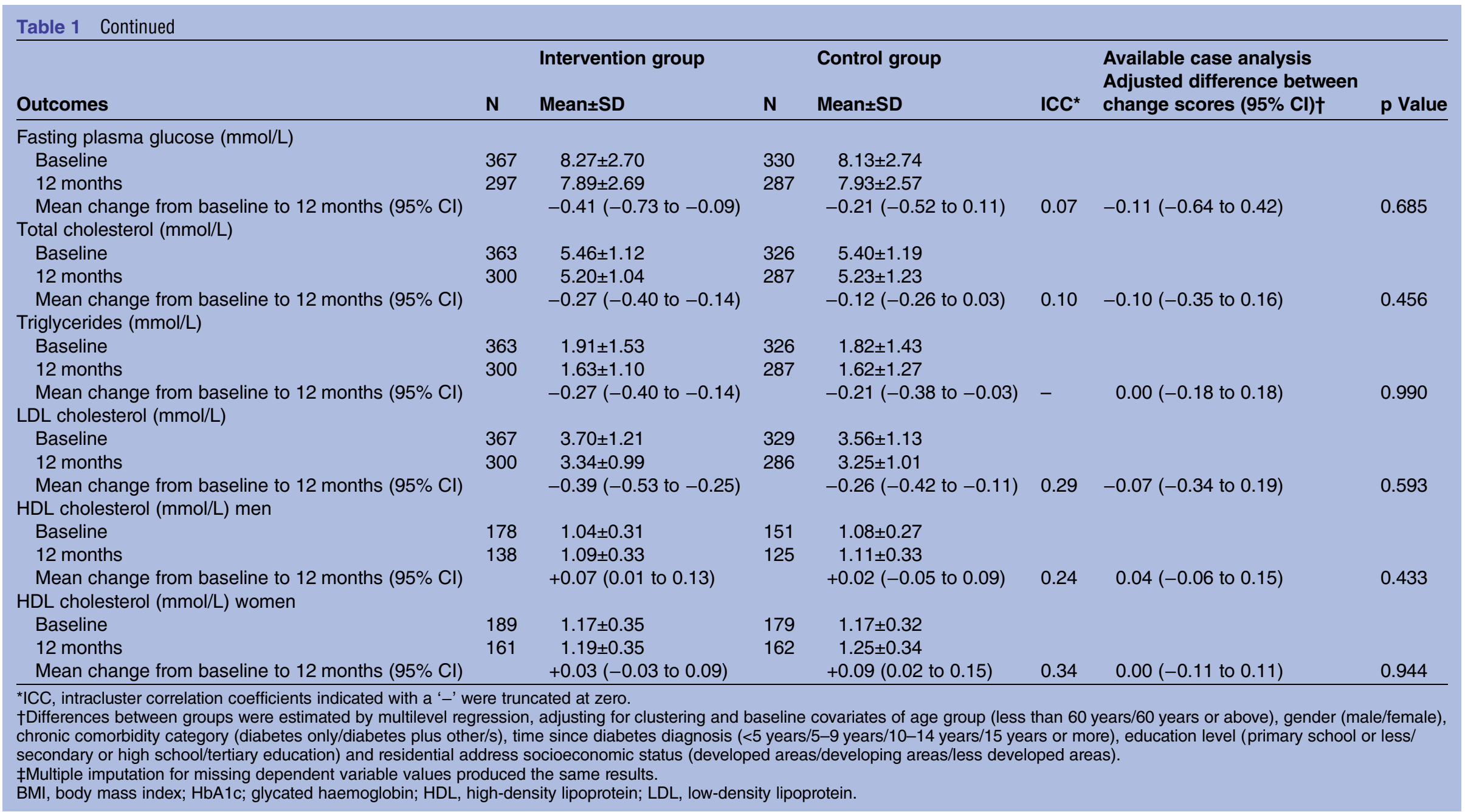


for missing data and then re-running the analyses. For each dependent variable with missing data, multiple imputation using multivariate regression with factors of intervention group and time produced five estimates.

\section{RESULTS}

Figure 1 depicts the flow of CHSs and participants through the trial. Of the original 41 randomised CHSs (21 intervention CHS and 20 control CHS), 2 control CHSs did not follow study protocol with regard to participant recruitment and baseline data collectionwhich resulted in incorrect ID allocation-and were removed from analysis (23 participants). This resulted in 21 intervention and 18 control CHSs included in the study.

In line with the recommendations for the reporting of pragmatic trials, numbers of eligible patients and numbers of those who were contacted and declined (along with the reasons for non-participation) were intended to be reported on; however, incomplete recruitment records in a considerable number of CHSs prevented this from occurring.

With regard to the 711 participants (intervention: $\mathrm{n}=372$; control: $\mathrm{n}=339$ ) who completed baseline data collection, $60(16.1 \%)$ and $43(12.7 \%)$ individuals from the intervention CHSs and control CHSs, respectively, withdrew throughout the study period. As a result, 312 and 296 participants in the intervention and control groups, respectively, were included in the analysis at 12 months (figure 1). Missing data analysis showed that dropouts were missing completely at random (Little's MCAR test: $\chi^{2}=19.4, \quad \mathrm{df}=18, \mathrm{p}=0.4$ ) when including variables of primary outcome, group allocation (intervention/ control) and baseline characteristics, as listed in table 3.

Table 3 shows the baseline characteristics of participants. Mean age was $63.8 \pm 8.3$ years and there were more women in the sample $(52.7 \%, 375 / 711)$ than men $(47.3 \%, 336 / 711)$. The majority were married $(89.8 \%$, $637 / 709)$, had received high school education $(68.7 \%$, $488 / 710)$ and were retired $(92.1 \%, 652 / 708)$. The mean duration of T2DM was $9.8 \pm 6.6$ years and approximately one-third of the sample was prescribed insulin at baseline $(31.5 \%, 223 / 708)$. Comorbid chronic conditions were present for the majority of participants $(82.1 \%, 584 / 711)$ and approximately one-fifth of the sample was classified as current smokers $(19.3 \%, 133 / 688)$. The intervention and control groups were similar for all variables at baseline, and even if statistical differences between groups were observed then the analysis method could have accounted for this by adjusting for baseline scores.

Table 1 shows the primary and secondary clinical outcomes, and table 2 the secondary psychosocial and selfcare outcomes, at baseline and 12 months by study group (figure 3A-D summarise the significant results). Outcome data at 6 months are detailed in online supplementary table S1 (clinical data) and online supplementary table S2 (psychosocial and self-care data).
At 12 months, no differential treatment effect was found for HbAlc (adjusted difference 0.02, 95\% CI -0.40 to $0.44, \mathrm{p}=0.929$ ), with treatment and control groups both showing significant improvements (Intervention: mean change $-3.86,95 \%$ CI -4.16 to -3.56 ; Control: mean change -3.69 , $95 \%$ CI -3.93 to -3.44). However, treatment effects showing significant improvement in intervention participants compared to controls were evident in systolic BP (adjusted difference $-3.57,95 \%$ CI -6.08 to $-1.05, \mathrm{p}=0.005$ ), waist circumference in men (adjusted difference $-1.28,95 \%$ CI -2.46 to $-0.11, \mathrm{p}=0.032$ ) and SDSCA-Specific Diet (adjusted difference $0.47,95 \%$ CI 0.13 to $0.80, \quad \mathrm{p}=0.006$. Psychological distress also significantly worsened in the control group compared to the intervention group (adjusted difference $-2.38,95 \%$ CI -4.64 to -0.12 , $\mathrm{p}=0.039$ ) (figure 3A). Other significant trends consistent with increasing weight gain in control participants were evident in the hip circumference increase in men, and increases in weight and BMI for men and for women. A significant increase in diastolic BP was also evident in controls only (mean change $+1.04,95 \%$ CI 0.00 to 2.08 ) (figure $3 \mathrm{~B}$ ). Interestingly, both groups also displayed significant improvements at 12 months in triglycerides (Intervention: mean change $-0.27,95 \%$ CI -0.40 to -0.14 ; Control: mean change $-0.21,95 \%$ CI -0.38 to -0.03); LDL cholesterol (Intervention: mean change $-0.39,95 \%$ CI -0.53 to -0.25 ; Control: mean change $-0.26,95 \%$ CI -0.42 to -0.11 ); and HDL cholesterol (Men-Intervention: mean change +0.07 , 95\% CI 0.01 to 0.13 ; Control: mean change $+0.02,95 \%$ CI -0.05 to 0.09: Women-Intervention: mean change +0.03 , $95 \%$ CI -0.03 to 0.09 ; Control: mean change $+0.09,95 \%$ CI 0.02 to 0.15 ; figure $3 \mathrm{C}$ ).

With regard to psychosocial and self-care outcomes at 12 months, a significant improvement was observed among intervention participants for SDSCA-Blood Glucose Monitoring (mean change +0.87 , 95\% CI 0.56 to 1.18) and SDSCA-Foot Care (mean change +0.46 , $95 \%$ CI 0.09 to 0.83 ), while control participants displayed a significant worsening in DM self-efficacy (mean change $-6.78,95 \%$ CI -11.95 to -1.62 ). The findings for QoL domains were variable: a significant worsening was observed among intervention participants for QoL-Physical (mean change $-2.28,95 \%$ CI -3.97 to -0.58 ) and among control participants for QoL-Social Relationship (mean change $-2.20,95 \%$ CI -4.25 to -0.16); while QoL-Psychological significantly worsened among both groups (Intervention: mean change -5.97 , $95 \%$ CI -8.14 to -3.79 ; Control: mean change -4.31 , $95 \%$ CI -6.69 to -1.94 ) (figure $3 \mathrm{D}$ ).

\section{Sensitivity analyses}

Sensitivity analyses involved generating five imputations for each bit of missing data from the dependent variables. The numbers of bits of participant data for each dependent variable that were available for the complete case analysis are shown in tables 1 and 2. The amount of 
Table 2 Psychosocial and self-care outcomes at baseline, and 12 months, by study group

\begin{tabular}{|c|c|c|c|c|c|c|c|}
\hline Outcomes & $\mathbf{N}$ & $\begin{array}{l}\text { Intervention group } \\
\text { Mean } \pm \text { SD }\end{array}$ & $\mathbf{N}$ & $\begin{array}{l}\text { Control group } \\
\text { Mean } \pm \text { SD }\end{array}$ & ICC* & $\begin{array}{l}\text { Available case analysis } \\
\text { Adjusted difference between } \\
\text { change scores }(95 \% \mathrm{Cl})^{\star}\end{array}$ & p Value \\
\hline \multicolumn{8}{|l|}{ Psychological distress (K10) } \\
\hline Baseline & 362 & $15.31 \pm 6.85$ & 328 & $14.97 \pm 6.24$ & & & \\
\hline 12 months & 305 & $16.16 \pm 6.44$ & 288 & $17.45 \pm 8.12$ & & & \\
\hline Mean change from baseline to 12 months (95\% Cl) & & $+0.80(-0.16$ to 1.77$)$ & & $+2.47(1.39$ to 3.55$)$ & 0.11 & $-2.38(-4.64$ to -0.12$)$ & $0.039 \dagger$ \\
\hline \multicolumn{8}{|l|}{ Diabetes management self-efficacy (CDMSES) } \\
\hline Baseline & 366 & $159.32 \pm 32.99$ & 334 & $158.96 \pm 34.85$ & & & \\
\hline 12 months & 306 & $156.72 \pm 32.68$ & 291 & $152.75 \pm 39.48$ & & & \\
\hline Mean change from baseline to 12 months (95\% Cl) & & $-2.61(-6.91$ to 1.69$)$ & & $-6.78(-11.95$ to -1.62$)$ & 0.26 & $10.71(-8.72$ to 30.13$)$ & 0.280 \\
\hline \multicolumn{8}{|l|}{ Diabetes self-care (SDSCA) } \\
\hline \multicolumn{8}{|l|}{ General diet } \\
\hline Baseline & 368 & $5.37 \pm 1.80$ & 337 & $5.44 \pm 1.85$ & & & \\
\hline 12 months & 305 & $5.34 \pm 1.50$ & 287 & $5.39 \pm 1.56$ & & & \\
\hline Mean change from baseline to 12 months $(95 \% \mathrm{Cl})$ & & $+0.04(-0.21$ to 0.29$)$ & & $-0.14(-0.39$ to 0.10$)$ & 0.22 & $0.15(-0.52$ to 0.82$)$ & 0.665 \\
\hline \multicolumn{8}{|l|}{ Specific diet } \\
\hline Baseline & 368 & $4.10 \pm 1.51$ & 337 & $4.37 \pm 1.62$ & & & \\
\hline 12 months & 305 & $4.41 \pm 1.24$ & 287 & $3.93 \pm 1.30$ & & & \\
\hline Mean change from baseline to 12 months (95\% Cl) & & $+0.34(0.14$ to 0.55$)$ & & $-0.49(-0.73$ to -0.25$)$ & 0.15 & $0.47(0.13$ to 0.80$)$ & 0.006 \\
\hline \multicolumn{8}{|l|}{ Exercise } \\
\hline Baseline & 369 & $5.27 \pm 2.07$ & 337 & $5.01 \pm 2.13$ & & & \\
\hline 12 months & 305 & $5.07 \pm 1.73$ & 287 & $4.94 \pm 2.11$ & & & \\
\hline Mean change from baseline to 12 months $(95 \% \mathrm{Cl})$ & & $-0.20(-0.46$ to 0.07$)$ & & $-0.11(-0.42$ to 0.21$)$ & 0.15 & $-0.12(-0.78$ to 0.54$)$ & 0.721 \\
\hline \multicolumn{8}{|l|}{ Blood glucose monitoring } \\
\hline Baseline & 366 & $1.46 \pm 1.75$ & 336 & $1.85 \pm 1.83$ & & & \\
\hline 12 months & 305 & $2.45 \pm 2.00$ & 287 & $1.88 \pm 1.62$ & & & \\
\hline Mean change from baseline to 12 months $(95 \% \mathrm{Cl})$ & & $+0.87(0.56$ to 1.18$)$ & & $+0.01(-0.24$ to 0.27$)$ & 0.21 & $0.43(-0.26$ to 1.11$)$ & 0.220 \\
\hline \multicolumn{8}{|l|}{ Foot care } \\
\hline Baseline & 368 & $4.50 \pm 2.79$ & 337 & $4.46 \pm 2.62$ & & & \\
\hline 12 months & 305 & $4.90 \pm 2.31$ & 287 & $4.58 \pm 2.51$ & & & \\
\hline Mean change from baseline to 12 months $(95 \% \mathrm{Cl})$ & & $+0.46(0.09$ to 0.83$)$ & & $+0.07(-0.30$ to 0.44$)$ & 0.10 & $0.55(-0.30$ to 1.39$)$ & 0.205 \\
\hline \multicolumn{8}{|l|}{ Quality of life (WHOQoL-BREF) } \\
\hline \multicolumn{8}{|l|}{ Physical domain } \\
\hline Baseline & 368 & $62.62 \pm 12.92$ & 338 & $63.31 \pm 13.99$ & & & \\
\hline 12 months & 306 & $60.35 \pm 12.21$ & 291 & $61.41 \pm 14.45$ & & & \\
\hline Mean change from baseline to 12 months $(95 \% \mathrm{Cl})$ & & $-2.28(-3.97$ to -0.58$)$ & & $-1.87(-3.84$ to 0.10$)$ & 0.10 & $1.18(-3.25$ to 5.61$)$ & 0.601 \\
\hline \multicolumn{8}{|l|}{ Psychological domain } \\
\hline Baseline & 368 & $68.87 \pm 14.45$ & 336 & $67.90 \pm 16.18$ & & & \\
\hline 12 months & 306 & $62.56 \pm 15.27$ & 290 & $63.70 \pm 15.50$ & & & \\
\hline
\end{tabular}




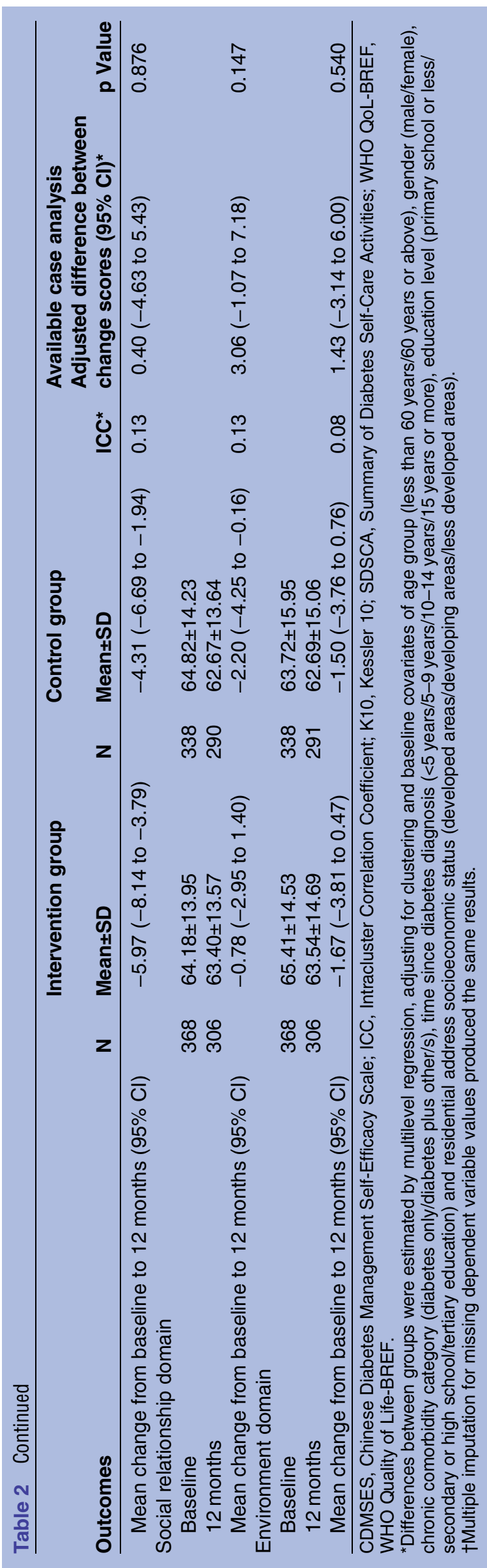

missing data for the dependent variables from the 711 participants (372 intervention group and 339 control group) was as high as $23.8 \%$ for the primary outcome of HbAlc at 6 months; $20.3 \%$ for psychological distress at 6 months; $23.5 \%$ for waist circumference for men at 12 months; $18.4 \%$ for systolic BP at 12 months; and $18.9 \%$ for SDSCA-Specific Diet at 12 months.

In the four significant treatment effects seen in the complete case analyses at 12 months, sensitivity analyses showed consistent results with two of these: waist circumference and SDSCA-Specific Diet at 12 months were not significant in the sensitivity analysis. Psychological distress and systolic BP remained significant in the sensitivity analyses, providing support that these outcomes were significantly better in the intervention group than in controls.

\section{DISCUSSION}

This pragmatic cluster RCT implemented in CHSs in urban China found no differential treatment effect in the primary outcome measure, HbAlc, with intervention and control groups both improving significantly. However, significant treatment effects favouring the intervention group were evident in psychological distress, systolic BP, waist circumference in men and SDSCA-Specific Diet. Two of these outcomes (psychological distress and systolic BP) were robust outcomes consistent with significant treatment effects, as supported in sensitivity analyses.

In addition to being statistically significant, the greater psychological distress observed in the control group compared with that in the intervention group is of clinical significance. The shift in the mean score for the control group at baseline from $14.97 \pm 6.24$ to $17.45 \pm 8.12$ at 12 months translates clinically to a shift in the mean from 'low risk of psychological distress' (scores 10-15) to 'moderate risk of psychological distress' (16-21). ${ }^{27}$ This result may be attributed to control group participants becoming more aware and knowledgeable about the degree of their T2DM condition and any comorbidities simply by participating in the study; and this greater awareness may have resulted in poorer well-being scores because control group participants were not given the same level of psychological support that was provided to intervention participants. This result may suggest that health intervention studies with older people in China may cause additional distress in participants if implemented without accompanying support.

The improvement in systolic BP observed in the intervention group (mean change $1.57 \mathrm{~mm} \mathrm{Hg}$ ) has the potential, if sustained, for reductions in deaths attributable to cardiovascular disease. In a meta-analysis of 61 prospective observational studies, Lewington et $a l^{28}$ (2002) found that even a $2 \mathrm{~mm} \mathrm{Hg}$ lower systolic BP would lead to approximately $10 \%$ lower stroke mortality and approximately 7\% lower mortality from ischaemic heart disease or other vascular causes. 
Table 3 Baseline characteristics of participants by study group

\begin{tabular}{llll}
\hline Baseline characteristics & Intervention group & Control group & Total \\
\hline Participants, $\mathrm{n}(\%):$ & $372(52.3)$ & $339(47.7)$ & 711 \\
Age in years, mean \pm SD & $63.7 \pm 7.6$ & $64.0 \pm 9.0$ & $63.8 \pm 8.3$ \\
Female, $\mathrm{n}(\%):$ & $191 / 372(51.3)$ & $184 / 339(54.3)$ & $375 / 711(52.7)$ \\
Married (including de facto), $\mathrm{n}(\%):$ & $338 / 371(91.1)$ & $299 / 338(88.5)$ & $637 / 709(89.8)$ \\
Retired, $\mathrm{n}(\%):$ & $343 / 370(92.7)$ & $309 / 338(91.4)$ & $652 / 708(92.1)$ \\
Secondary/high school education, n (\%): & $266 / 372(71.5)$ & $222 / 338(65.7)$ & $488 / 710(68.7)$ \\
Duration of T2DM in years, mean \pm SD & $10.0 \pm 6.5$ & $9.6 \pm 6.6$ & $9.8 \pm 6.6$ \\
Currently prescribed insulin, $\mathrm{n}(\%):$ & $124 / 371(33.4)$ & $99 / 337(29.4)$ & $223 / 708(31.5)$ \\
Comorbid conditions present, $\mathrm{n}(\%):$ & $316 / 372(84.9)$ & $268 / 339(79.1)$ & $584 / 711(82.1)$ \\
Current Smoker, $\mathrm{n}(\%):$ & $68 / 367(18.5)$ & $65 / 321(20.2)$ & $133 / 688(19.3)$ \\
\hline T2DM, type 2 diabetes mellitus. & & &
\end{tabular}

Other significant trends over the 12-month period consistent with increasing weight gain in control subjects were evident in the waist and hip circumference increases in men, and increases in weight and BMI for men and for women. This suggests that the MI intervention may have been influential in preventing weight gain, especially in men, since weight-related measures had significantly increased in control subjects only. A significant increase in diastolic BP was also evident in controls only, with the associated consequences being increased risk of cardiovascular disease.

Although glycaemic control did not differentially improve, HbAlc in both groups changed significantly and for the better, as did triglycerides, LDL cholesterol and HDL cholesterol. The mean HbAlc at baseline of $>10 \%$ in both groups suggests that participants in our sample were either not accessing CHSs for T2DM management or were receiving suboptimal T2DM care prior to enrolment in this trial. Accurate health service utilisation records were not able to be obtained for the period before our study; however, it can be assumed that this trial served as a catalyst for the revitalisation of primary care delivery to individuals with T2DM. While the possible revitalisation may be attributed to a multitude of practice, participant and study-related factors, the interpretation of the true effect of MI is consequently limited.

The outcome measures of HbAlc and fasting plasma glucose typically correlate very reliably with each other in the management of T2DM, yet this was not the case in the present trial. Owing to the complex relationship between the two, we are unable to accurately explain why the change in HbAlc was not reflected in fasting plasma glucose in this study. We can exclude the possibility of non-fasting blood being sampled as the fasting duration was documented at each health check.

The significant improvement of both treatment groups for HbA1c and numerous secondary outcomes may partly be explained by the regular clinical testing and subsequent feedback to control group participants following each clinical health check at baseline, 6 and 12 months. The majority of patients with T2DM in China do not regularly monitor their blood glucose nor do they attend regular appointments specifically to manage their T2DM. According to $\mathrm{Xu}$ et $a l^{29}$ in a convenience sample of 201 Chinese adults with T2DM, it was found that only $4.5 \%$ of participants performed selfmonitoring of blood glucose on a daily basis. Furthermore, a more recent study investigating treatment-seeking rates among individuals with DM reported that only $25.8 \%$ received treatment for DM and, of those, only $39.7 \%$ had adequate glycaemic control. ${ }^{2}$ Additionally, the Diabcare-Asia (China) study in 2003 found that only $50 \%$ of patients with diagnosed DM had a HbAlc measurement in the previous 12 months, ${ }^{5}$ despite guidelines recommending HbAlc to be assessed annually. ${ }^{7}$ By informing individuals of their health status in relation to T2DM, it may have prompted individuals and their CHS care providers to be more focused on their condition than otherwise expected, thus altering the 'usual care' condition. Additionally, to maximise participation, control group and intervention group participants both received free clinical health checks as part of their participation in the study, further altering the 'usual care' condition. Possible consequences of these alterations to usual care may have been an improvement in adherence to the T2DM self-care regimen and increased attendance at CHS appointments; as well as the start of medication for the treatment of uncontrolled variables. While these consequences may have contributed to the significant improvement in glycaemic control among control group participants, intervention participants would equally have benefited from the feedback of clinical health checks. One important outcome of the alteration of the 'usual care' condition and the result of intervention and control groups both displaying significant improvements in glycaemic control is that it provides evidence to support the development of a national T2DM monitoring service that provides free clinical health checks to patients with T2DM at more regular intervals those currently recommended.

A further explanation for the significant improvement in HbAlc and numerous secondary outcomes among both treatment groups is the possible contamination between CHSs. While every attempt was made to avoid 


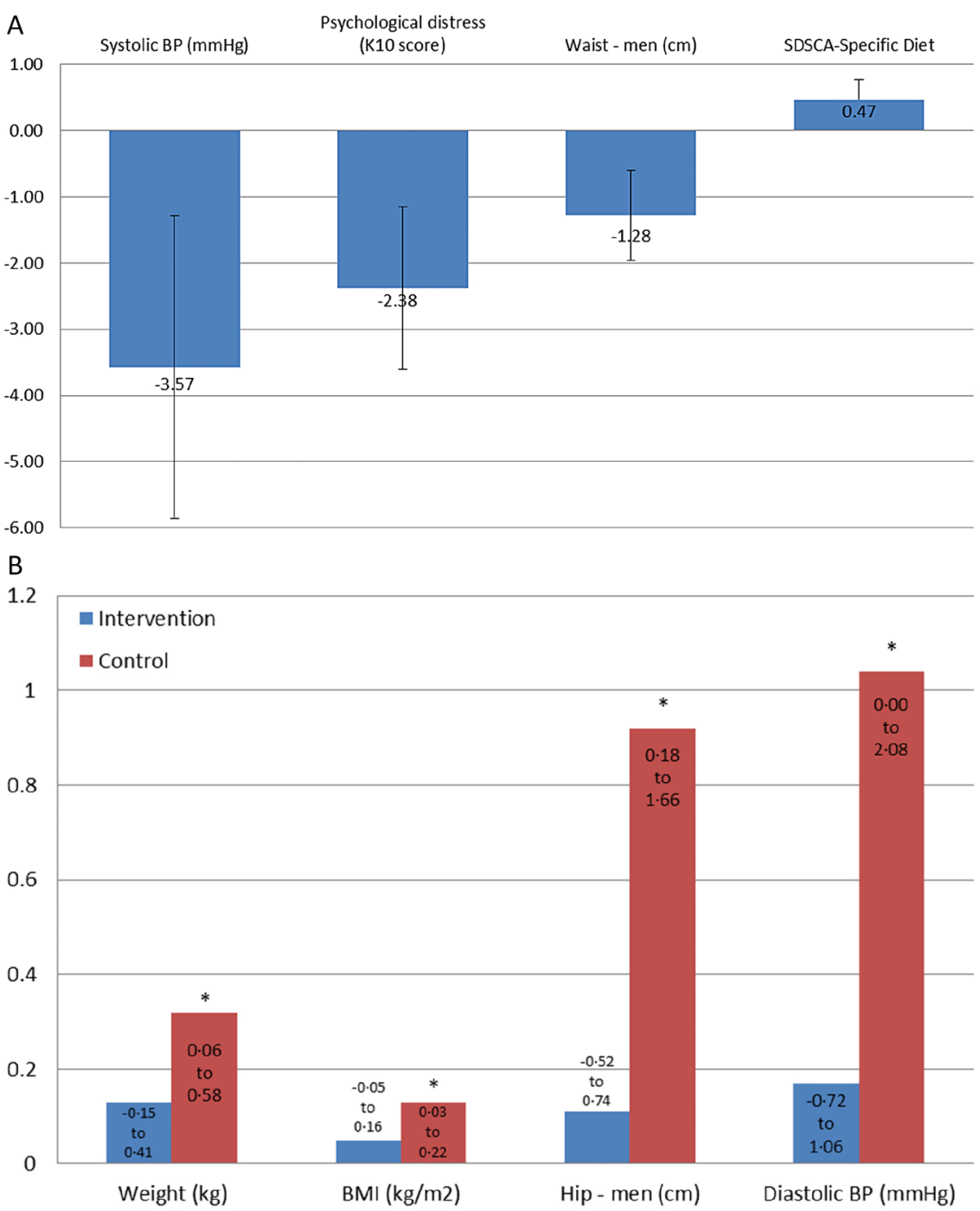

Figure 3 Summary of significant study findings. (A) Significant treatment effects shown for the 12-month change scores. Negative scores indicate better results in the intervention group (except SDSCA-Specific Diet, where positive scores are better). Waist circumference and SDSCA-specific diet change scores were not significant between treatment and controls in the sensitive analyses, suggesting that they were not robust outcomes. Not shown are significant changes at 6 months for WHO QOL-BREF (environment) and SDSCA-general diet (see online supplementary table). (B) Changes in clinical scores at 12 months compared to baseline. *Significant changes indicating poorer scores in control group only. (C) Changes in clinical scores at 12 months compared to baseline. *Significant changes indicating better scores. Not shown are significant improvements in fasting plasma glucose (FPG) and total cholesterol (TC) in the controls at 6 months (see online supplementary table S1). (D) Changes in scores of psychosocial and self-care measures at 12 months compared to baseline. Positive scores indicate better scores on scales. Not shown are significant changes in C-DMSES, SDSCA-SpD, SDSCA-Ex, WHO QOL-BREF (physical, psychological, social relationship) at 6 months (see online supplementary table S2). BMI, body mass index; CDMSES, Chinese Diabetes Management Self-Efficacy Scale; HbA1c, glycated haemoglobin; HDL, high-density lipoprotein; LDL, low-density lipoprotein; SDSCA-SpD, Summary of Diabetes Self-Care Activities-specific diet; Systolic BP, systolic blood pressure; TG, triglycerides; WHO QoL-BREF, WHO Quality of Life-BREF.

contamination between CHSs, the study received a considerable amount of media attention throughout the intervention phase, which may have resulted in participants and CHS staff altering their usual behaviour. Additionally, all CHSs participating in this study are managed and administered by the same government health bureau, which may also have affected the delivery of the intervention and control procedures. This was a pragmatic trial, and the above factors would only have increased the inherited heterogeneity and dilution of any treatment effects in the intervention group that are known to occur in pragmatic trials. ${ }^{30} 31$

Other than the findings of our pilot study, ${ }^{32}$ no cluster RCTs have assessed the effectiveness of health coaching based on MI principles for the management of patients with T2DM in China. Although our pilot study utilised the same pragmatic design as the present trial, some notable differences in the results for HbAlc were 


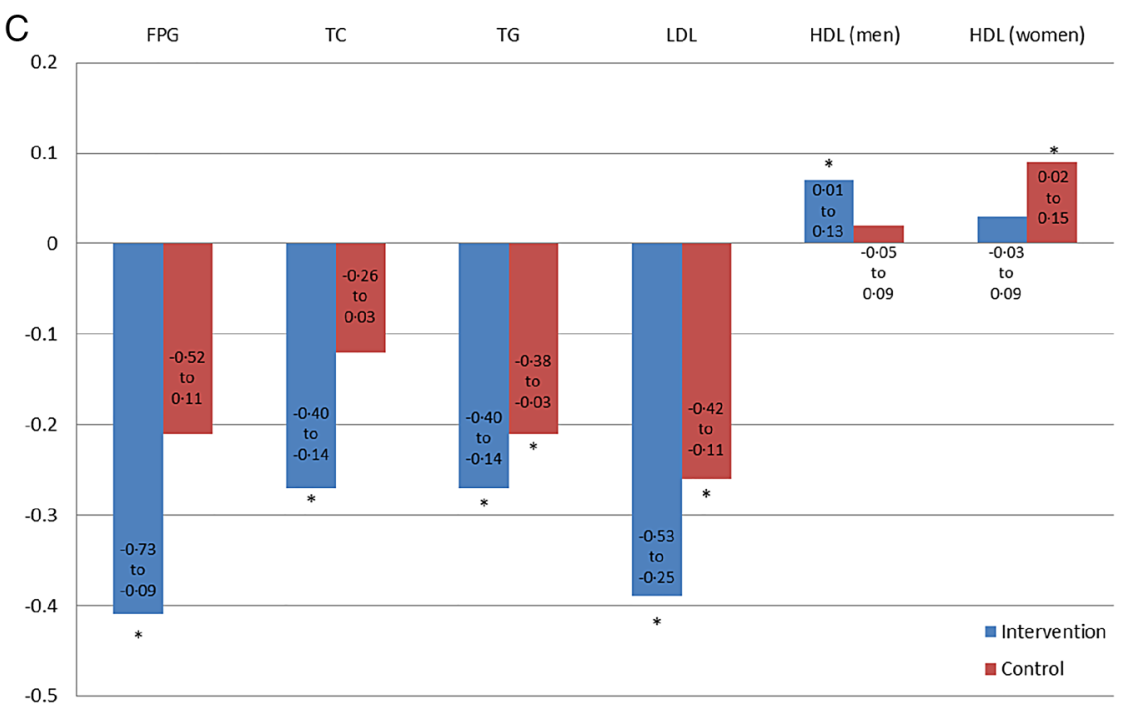

HbA1c

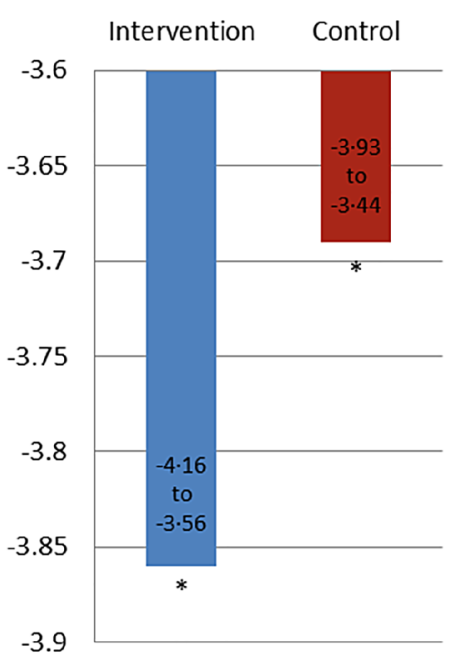

$\mathrm{D}_{1}$

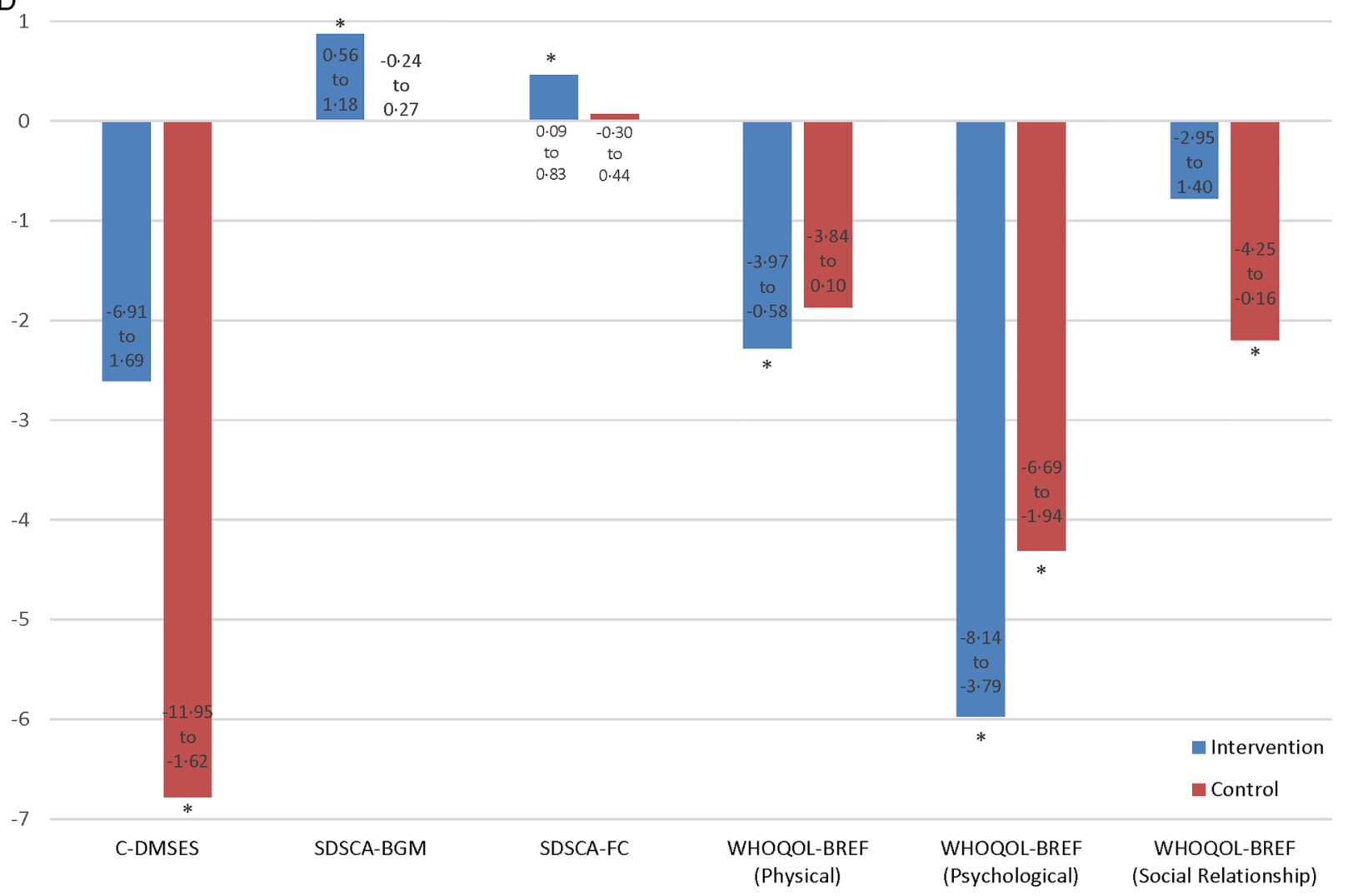

Figure 3 Continued

observed when comparing trials. In the pilot study, a significant improvement in HbAlc from baseline to 6 months was observed in the intervention group only. Additionally, the baseline HbAlc among both treatment groups in the pilot study was substantially lower (approximately $7.0 \%$ ) than that observed in the present trial (approximately 10\%). One explanation for this variation may be the difference in the quality of care delivered by the CHSs, between the pilot and the present trial. The pilot study was conducted in the Fangzhuang Community Health Centre, which was the only nationally certified centre in Fengtai district at the time of both studies. ${ }^{33}$ As such, it is possible that the usual care provided in the pilot to individuals with T2DM was of higher quality than that offered by the CHSs in the present trial. Another possibility is that, in the pilot compared to the full trial, less 'leakage' occurred across the treatment and control arms of the study. The study was widely reported across the Chinese media and this may have impacted on treatment fidelity in the control arm of the full study.

Our findings are consistent with a recent cluster RCT that similarly found lifestyle counselling based on MI 
principles did not differentially improve HbAlc, nor did it have an impact on the majority of secondary outcomes assessed in our study. ${ }^{34}$ Some research exists in China supporting the use of MI for the improvement of glycaemic control in patients with T2DM, however, outcomes were assessed at 6 months and the methodological quality of studies was variable. ${ }^{35}$ A number of international traditional RCTs ${ }^{36-38}$ have also been published that corroborate the findings of the present research, in particular the trial by Rubak et al, ${ }^{36}$ which found significant improvements in metabolic status within both treatment groups at 12 months with no difference found when comparing groups. However, no previous studies have observed improvements in HbA1c of the same magnitude as the present trial. The baseline HbAlc values of these studies were substantially lower (approximately 7\%), consequently limiting their potential for improvement.

The key strength of this study is that it was a pragmatic trial, specifically tailored to be implemented in real world urban Chinese CHSs that utilised existing resources, hence maximising external validity. Additionally, the cluster RCT design minimised contamination between CHSs, and the stratification and randomisation procedure of both CHSs and participants minimised selection bias, and increased the generalisability of results to other populations of patients with T2DM in urban China. Indeed, the baseline demographic and clinical characteristics of our sample were similar to those previously observed in individuals with DM who live in urban and developed regions in China. ${ }^{2}$ For example, in our sample, there were approximately similar proportions of males and females; the mean age of our sample (64 years) was within the second highest population prevalence age band for DM (slightly higher population prevalence for $\geq 70$ years); participants were more likely to have education levels of secondary/high school or higher, and were also more likely to be overweight, and have elevated total cholesterol, LDL cholesterol and triglyceride levels.

The differential treatment effects in intervention participants receiving MI compared with controls is a promising finding, and provides evidence for continuing such interventions. While it is noted that the magnitude of average improvements was small, this may be attributed to the pragmatic nature of the trial. The wide eligibility criteria utilised contributed to a broad population of individuals with T2DM that more closely resembles that observed in typical clinical practice; and this variability between participants may have potentially diluted the observed treatment effects. ${ }^{30} 31$ Additionally, the potential variability of intervention delivery between each CHS, as well as some possible contamination of the control CHSs, may have further contributed to the probable dilution of treatment effect. If this study had adopted a more traditional, explanatory RCT design under more experimental conditions, it is possible that the observed treatment effects may have been larger.
A significant limitation to this study is that information on medication use and adherence was not assessed rigorously enough. While it could be assumed that both groups would have been medicated similarly as outlined in the Chinese Guideline for Diabetes Prevention and Management, ${ }^{7}$ it was not possible to accurately measure the impact of any change in medication use throughout the trial. A further limitation is that intervention fidelity has not as yet been assessed; therefore it has not been possible to distinguish between participants with regard to quality of MI received. All coaching sessions were audio recorded and a subsequent publication is planned that will utilise the Motivational Interviewing Treatment Integrity (MITI) framework to assess intervention integrity. Additionally, the pragmatic nature of this study meant that data collection was conducted by multiple data collectors from multiple CHSs. Despite all data collectors being trained according to the data collection protocol, quality of data varied and this resulted in higher than anticipated levels of missing data. Further, the lack of blinding of outcome assessors as well as the limitations inherent in self-reported data are worth noting when interpreting the findings. Another limitation is that only small differential effects in some secondary measures were detected, and although small effects in pragmatic trials are common, since all sources of variance cannot be controlled, it is possible that they result from type I errors. Multiple examinations also increase risk of type I errors.

The results of this pragmatic cluster RCT provides some evidence for the utility of training health professionals in the use of MI in the management of patients with T2DM within Chinese CHSs. We note that the health professionals in this trial were trained in patientcentred behavioural counselling and MI, which differs from their usual, somewhat authoritarian method of interacting with patients. While the health coaches embraced the new approach very well, the training comprised $20 \mathrm{~h}$ and future studies in China could assess how much training is needed to produce competent MI health coaches. It is possible that in the current trial the health coaches had not reached a sufficient standard to be effective MI counsellors despite improving their skills. The aforementioned assessment of treatment integrity will investigate this further.

\section{CONCLUSION}

The coaching approach employed in this study is entirely consistent with China's primary healthcare reforms. T2DM is one of China's most prevalent and burdensome chronic illnesses, and one that is amenable to effective community-based primary healthcare interventions. Differential treatment effects were observed neither for HbA1c nor for the majority of secondary outcomes, however, significant changes within both groups with regard to numerous clinical outcomes, including HbAlc, highlight the advantages of regular, 
free clinical health checks for patients with T2DM. With a strong focus on self-management and health coach support, this study has the potential to be adapted to other chronic diseases, as well as to other regions of China. Indeed, an extension of the present trial is planned for implementation in Shenzhen in 2016/17, as part of the function of the newly formed International Primary Health Care Research Institute.

\section{Author affiliations}

${ }^{1}$ RDNS Institute, Melbourne, Victoria, Australia

${ }^{2}$ Faculty of Medicine Nursing and Health Sciences, School of Primary Health Care, Monash University, Melbourne, Victoria, Australia

${ }^{3}$ International Institute for Primary Health Care Research, Shenzhen, China

${ }^{4}$ Beijing Office for Cancer Prevention and Control, Peking University Cancer Hospital and Institute, Beijing, China

${ }^{5}$ School of Public Health, Peking University Health Science Centre, Beijing, China

${ }^{6}$ School of Clinical Sciences, Monash University, Melbourne, Victoria, Australia

Acknowledgements The authors thank Professor Liang Wannian, formal executive director of Beijing Health Bureau; Zhang Yang and Dr Xiao Lixin of the Health Bureau of Fengtai Government; Yang Zhiyun and Dr Zhouwei of Fengtai Community Health Management Centre; and all the directors and health coaches from the 41 Community Health Stations who participated in this trial.

Contributors CB and ST led the conception of the study. CB, ST and TZ obtained research and operational funding, and HY participated. AC, CB and HY led the design of the study protocol. AC, CB, SAT and JCE drafted the article and all the authors contributed to critical revision. SL, AC, HY and TZ coordinated the data collection and data cleaning. JCE led the statistical analysis of the data and $\mathrm{AC}$ contributed. All the authors participated in the interpretation of the trial data. All the authors, internal and external, had full access to the study data, and take responsibility for the integrity of the data and the accuracy of the data analysis. All the authors have approved the final version of the manuscript. CB is the guarantor.

Funding This trial was funded by the Fengtai Health Bureau, Beijing, China, and in kind support was provided by Monash University, Australia, and Peking University, China. The funders of the study had no role in study design, data collection, data analysis, data interpretation, writing of the report, or decision to submit for publication. The views and opinions expressed therein are those of the authors and do not necessarily reflect those of the funding body.

Competing interests All the authors have completed the ICMJE uniform disclosure form at http://www.icmje.org/coi_disclosure.pdf and declare: no support from any organisation for the submitted work; no financial relationships with any organisations that might have an interest in the submitted work in the previous 3 years; no other relationships or activities that could appear to have influenced the submitted work.

Ethics approval This study was approved by Monash University Human Research Ethics Committee (CF11/2657-2011001550) and all participants gave informed consent before taking part.

Provenance and peer review Not commissioned; externally peer reviewed.

Data sharing statement No additional data are available.

Open Access This is an Open Access article distributed in accordance with the Creative Commons Attribution Non Commercial (CC BY-NC 4.0) license, which permits others to distribute, remix, adapt, build upon this work noncommercially, and license their derivative works on different terms, provided the original work is properly cited and the use is non-commercial. See: http:// creativecommons.org/licenses/by-nc/4.0/

\section{REFERENCES}

1. International Diabetes Federation. IDF diabetes atlas. 7th edn Brussels, Belgium: International Diabetes Federation, 2015.
2. $\mathrm{Xu} \mathrm{Y}$, Wang L, He J, et al. Prevalence and control of diabetes in Chinese adults. JAMA 2013;310:948-59.

3. World Health Organization. Preventing chronic diseases: a vital investment: WHO global report. Geneva, Switzerland: World Health Organization, 2005

4. Chen Z. Launch of the health-care reform plan in China. Lancet 2009;373:1322-4

5. Pan C. Diabetes care in China: meeting the challenge. Diabetes Voice 2005;50:9-12.

6. International Diabetes Federation. China spends RMB 173.4 billion (US $\$ 25$ billion) a year on diabetes treatment. Secondary China spends RMB 173.4 billion (US $\$ 25$ billion) a year on diabetes treatment [Web Page]. 2010. http://www.idf.org/china-spendsrmb-1734-billion-us25-billion-year-diabetes-treatment

7. Chinese Diabetes Society. China's prevention and treatment guideline for type 2 diabetes Mellitus (2013 edition). Chin J Diab Mellitus 2014;6:447-98.

8. Ismail K, Winkley K, Rabe-Hesketh S. Systematic review and meta-analysis of randomised controlled trials of psychological interventions to improve glycaemic control in patients with type 2 diabetes. Lancet 2004;363:1589-97.

9. Zhu X, Gong Y, Yao S. [The effect of biofeedback assisted relaxation training on cytokines in patients with type II diabetes: a follow up study]. Chin J Clin Psychol 2001;9:5-10.

10. Huang X, Song L, Li T. [The effect of social support on type II diabetes with depression]. Chin J Clin Psychol 2001;9:187-9.

11. Fu M, Dong Y. [Effect of psychological intervention on depression symptoms and blood glucose level of patients with diabetes mellitus in China: a meta-analysis]. J Chin Gen Pract 2013;16:436-9.

12. Browning CJ, Thomas SA. Behavioural change: an evidence-based handbook for social and public health. Elsevier/Churchill Livingstone, 2005.

13. Miller WR, Rollnick S. Motivational interviewing: helping people change. 3rd edn. New York, NY: Guilford Press, 2012.

14. Browning C, Chapman A, Cowlishaw S, et al. The Happy Life $\mathrm{Club}^{\mathrm{TM}}$ study protocol: a cluster randomised controlled trial of a type 2 diabetes health coach intervention. BMC Public Health 2011;11:90.

15. Browning CJ, Yang $\mathrm{H}$, Zhang $\mathrm{T}$, et al. Implementing a chronic disease self-management program into China: Happy Life Club ${ }^{\mathrm{TM}}$. Front Public Health 2015:2:181.

16. Fengtai Bureau of Statistics. Current demography of Fengtai old residents. Secondary Current demography of Fengtai old residents, 2012. http://www.ft.bjstats.gov.cn/html/20130617/807522. $\mathrm{html}$

17. Loudon K, Treweek S, Sullivan F, et al. The PRECIS-2 tool: designing trials that are fit for purpose. BMJ 2015;350:h2147.

18. State Commission Office for Public Sector Reform. The guideline for facility establishment standards of urban community centers. Document No. 96, August 18; 2006 [in Chinese]

19. Campbell MK, Mollison J, Steen N, et al. Analysis of cluster randomized trials in primary care: a practical approach. Fam Pract 2000;17:192-6.

20. Kessler RC, Andrews G, Colpe LJ, et al. Short screening scales to monitor population prevalences and trends in non-specific psychological distress. Psychol Med 2002;32:959-76.

21. [No authors listed]. Development of the World Health Organization WHOQOL-BREF Quality of Life Assessment The WHOQOL Group. Psychol Med 1998:28:551-8.

22. Toobert DJ, Hampson SE, Glasgow RE. The summary of diabetes self-care activities measure: results from 7 studies and a revised scale. Diabetes Care 2000;23:943-50.

23. Vivienne Wu SF, Courtney M, Edwards $\mathrm{H}$, et al. Development and validation of the Chinese version of the Diabetes Management Self-efficacy Scale. Int J Nurs Stud 2008;45:534-42.

24. Perera R, Heneghan C, Yudkin P. Graphical method for depicting randomised trials of complex interventions. BMJ 2007;334:127-9.

25. Chinese Nutrition Society. Dietary Guidelines for Chinese Residents, Tibet People's Publishing House, 2007 [in Chinese].

26. Meng $\mathrm{Q}$, Fang $\mathrm{H}$, Liu $\mathrm{X}$, et al. Consolidating the social health insurance schemes in China: towards an equitable and efficient health system. Lancet 2015;386:1484-92.

27. Australian Bureau of Statistics. Information paper: Use of the Kessle Psychological Distress Scale in ABS Health Surveys, Australia, 2007-08. (cat no. 4817.0.55.001). Canberra, 2012. http://www.abs. gov.au/ausstats/abs@.nsf/mf/4817.0.55.001

28. Lewington S, Clarke R, Qizilbash N, et al. Age-specific relevance of usual blood pressure to vascular mortality: a meta-analysis of 
individual data for one million adults in 61 prospective studies. Lancet 2002;360:1903-13.

29. Xu Y, Toobert D, Savage C, et al. Factors influencing diabetes self-management in Chinese people with type 2 diabetes. Res Nurs Health 2008;31:613-25.

30. Patsopoulos NA. A pragmatic view on pragmatic trials. Dialogues Clin Neurosci 2011;13:217-24.

31. MacPherson H. Pragmatic clinical trials. Complement Ther Med 2004;12:136-40.

32. Browning CJ, Yang H, Zhang T, et al. Implementing a chronic disease self-management program into China: the Happy Life Club $^{\mathrm{TM}}$. Front Public Health 2015;2:181.

33. National Health and Family Planning Commission People's Republic of China. 2011 National Demonstration Community Health Service Center List. Secondary 2011 National Demonstration Community Health Service Center List, 2011. http://www.moh.gov.cn/zwgkzt/ wsbysj/201112/53666.shtml

34. Jansink R, Braspenning J, Keizer E, et al. No identifiable Hb1Ac or lifestyle change after a comprehensive diabetes programme including motivational interviewing: a cluster randomised trial. Scand $J$ Prim Health Care 2013;31:119-27.

35. Song D, Xu TZ, Sun QH. Effect of motivational interviewing on self-management in patients with type 2 diabetes mellitus: a meta-analysis. Int J Nurs Sci 2014;1:291-7.

36. Rubak S, Sandbæk A, Lauritzen T, et al. Effect of "motivational interviewing" on quality of care measures in screen detected type 2 diabetes patients: a one-year follow-up of an RCT, ADDITION Denmark. Scand J Prim Health Care 2011;29:92-8.

37. Heinrich E, Candel MJJM, Schaper NC, et al. Effect evaluation of a Motivational Interviewing based counselling strategy in diabetes care. Diabetes Res Clin Pract 2010;90:270-8.

38. Rosenbek Minet LK, Wagner L, Lønvig EM, et al. The effect of motivational interviewing on glycaemic control and perceived competence of diabetes self-management in patients with type 1 and type 2 diabetes mellitus after attending a group education programme: a randomised controlled trial. Diabetologia 2011;54:1620-9. 